\title{
Evidence for GAL3ST4 mutation as the potential cause of pectus excavatum
}

Cell Research (2012) 22:1712-1715. doi:10.1038/cr.2012.149; published online 13 November 2012

\section{Dear Editor,}

Pectus excavatum (PE) is the most common congenital deformity of chest wall that occurs in approximately 1 in every 400 births [1]. PE is characterized by the abnormal development of sternum and costal cartilage, which reduces the thoracic volume, restricts the pulmonary movement and leads to cardiac compression [1]. Without timely medical interventions, PE may become progressively worse during the adolescent growth spurt $[1,2]$. The etiology of PE remains largely undefined, but accumulating clinical evidence supports that PE is a genetic disorder that is either inherited dominantly or recessively as $\sim 40 \%$ of the patients have affected family members with similar congenital deformities [3]. However, few pathogenic genes have been identified for PE yet [2]. Thus, systematic analysis of the genetic and molecular mechanisms underlying PE is highly warranted.

In this study, we analyzed a four-generation Han Chinese family with PE that showed dominant inheritance (Figure 1A). Four of the family members (II:5, III:4, IV:1 and IV:2) were diagnosed as "cup-shaped" or "bowl-shaped" PE. To systematically screen for the candidate genes that are likely to be causative for PE in this pedigree, we sequenced the whole exomes for all the four affected members and one of the unaffected normal individuals (III:6) (Supplementary information, Data S1). We generated the sequencing reads with Illumina's next generation sequencing technology as described previously [4]. In total, we sequenced the exome sequences to a mean depth of $58 \times$ or greater for each individual, which covered $\sim 90 \%$ of the targeted bases, sufficiently for confident variant calling (Supplementary information, Table S1).

To sift out the potentially pathogenic mutations, we first filtered all the identified genetic variants that were shared by all the four patients against the list of variants obtained from the unaffected normal control (Supplementary information, Table S2). Through this way, we identified 771 case-specific genetic variants that were shared by all the affected individuals. Given that the incidence of PE is very low and that no racial difference in incidence has been reported, it is highly possible that mutations causative for familial PE should be absent from the general population. Therefore, we further filtered the case-specific variants against the sets of genetic polymorphisms from the dbSNP132 and the 1000 Genome Project databases (as of November 23, 2010). After these two steps, only three novel genetic variants remained as the candidate mutations that were likely linked to PE.

Of the three novel variants that were predicted to be related to PE in this family, two were annotated as missense mutations by the SIFT program (http://sift.jcvi. org/). We next orthogonally validated these two nonsynonymous variants in the source samples (II:5, III:4, III:6, IV:1 and IV:2) by Sanger sequencing and found that one was likely to be a false-positive mutation in the repetitive region of human genome (Supplementary information, Table S4). The other heterozygous mutation g.chr7: $99764688 \mathrm{G}>\mathrm{A}$ was confirmed to be shared by all the four patients but absent from the healthy family members with DNA samples available, including II:3, II:6 and III:6 who are genetically related members and III:5 who is genetically unrelated to the family (Figure 1B). These results provided genetic evidence that mutation g.chr7: $99764688 \mathrm{G}>\mathrm{A}$ was most likely to be pathogenic for PE in this family. We then genotyped this mutation in an additional cohort of 378 unrelated healthy individuals and further confirmed its potential causative role in PE pathogenesis as none of the normal controls showed mutation at this genomic site.

Mutation g.chr7: $99764688 \mathrm{G}>\mathrm{A}$ was predicted to affect the first exon of GAL3ST4 and result in a substitution of the positively-charged arginine (R) with the uncharged tryptophan (W) at the residue 11 of the encoded protein (c.424G $>$ A, p.R11W). Evolutionary conservation analysis revealed that the mutated residue 11 (R) of Gal3ST-4 is highly conserved across several vertebrate species (Figure 1C), which may indicate that this mutation is highly likely to disrupt the normal 
A

B

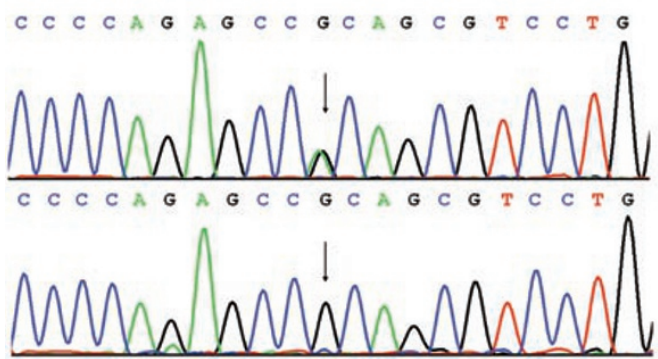

C

$\begin{array}{ll}\text { Human } & \text { MGPLSPARTLRLWGPRSLGV } \\ \text { Chimpanzee } & \text { MGPLSPARTLRLWGPRSLGV } \\ \text { Rhesus } & \text { MGPLSPARTLRLWGPRSLGV } \\ \text { Cow } & \text { MGVLSPTRTMRLWGPRSLGV } \\ \text { Rat } & \text { MGPLSPARTMRLWRPRSLGV } \\ \text { Mouse } & \text { MGPLSPARTMRLWRPRSLGV }\end{array}$

D

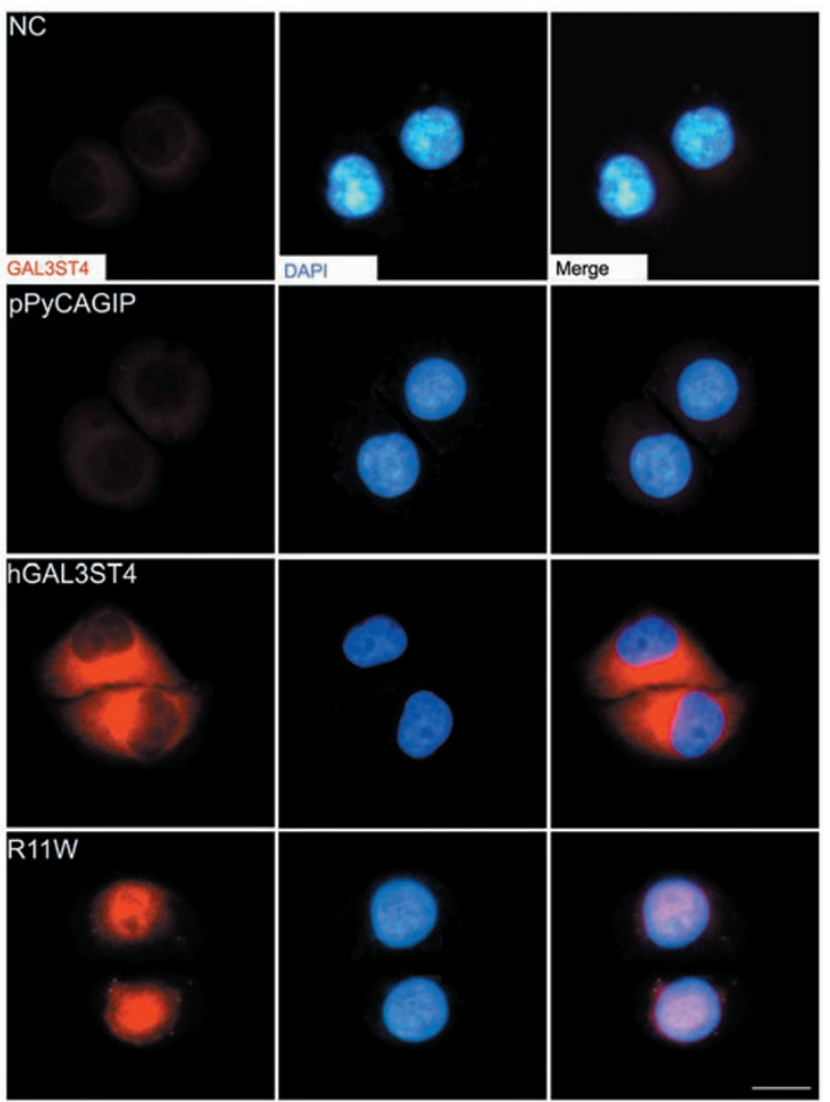

Figure 1 Genetic and molecular evidence for GAL3ST4 mutation as the cause of familial PE. (A) The pedigree structure of the family with congenital PE analyzed in this study. The black boxes represent the affected individuals. The arrow beside the black box represents the proband. (B) Chromatogram traces from Sanger sequencing showing the validated missense mutation g.chr7: $99764688 \mathrm{G}>\mathrm{A}$. The upper panel shows the sequencing result of the proband and the lower panel shows the sequencing result of III:6. (C) Evolutionary conservation analysis of the amino acid affected by the identified missense mutation. The affected amino acid is shown in the red box. (D) Immunolocalization analysis of wild-type and mutant GAL3ST4 in transfected HeLa cells. Red shows the anti-GAL3ST4 staining; blue shows artificial coloring of nuclei by DAPI. Cells were transfected with plasmid DNA (pPyCAGIP vector) encoding the wild-type (hGAL3ST4) and mutant GAL3ST4 (p.R11W), respectively. Normal HeLa cells (NC) and cells transfected with empty vector (pPyCAGIP) served as controls. Cells transfected with hGAL3ST4 showed GAL3ST4 expression in the cytoplasm, whereas the expression of p.R11W was detected as patches distributed over the entire nucleus.

functions of the protein. Gal3ST-4 is the fourth member of the Gal: $\rightarrow 3$-sulfotransferase family that catalyzes the C-3 sulfation of galactoses in O-linked glycoproteins [5]. It has been shown that sulfation of the glycan chains is associated with the specific physiological functions of the various glycoproteins. The Golgi apparatusassociated sulfotransferases carry out the sulfation of glycans by transferring the sulfate group from the phosphoadenosine-phosphosulfate (PAPS) to a precursor oligosaccharide [6]. The p.R11W mutation identified in our study is located in the signal peptide region of Gal3ST-4. Therefore, we reasoned that the substitution of arginine with tryptophan may greatly influence the functions of Gal3ST-4 by changing its subcellular localization.

To test this possibility, we expressed the mutant and wild-type forms of human GAL3ST4 in HeLa cells, which only express very low levels of endogenous 
GAL3ST4. The GAL3ST4 coding sequence was cloned into the pPyCAGIP expression vectors and the GAL3ST4 p.R11W mutant was generated by PCR (Supplementary information, Data S1 and Table S3). Our immunofluorescence analysis showed that the wild-type GAL3ST4 was predominantly detected in the cytoplasm whereas the mutant GAL3ST4 was mainly detected in the nucleus (Figure 1D).

Previous studies have established that sulfation of the proteoglycans is crucial for the normal development of cartilages and bones [6]. The major catalytic machinery responsible for the biosynthesis and breakage of sulfate esters in the proteoglycans is composed of various enzymes and transporters. Mutations in genes encoding the transmembrane transporters of sulfate or enzymes involved in the synthesis of PAPS have been identified as the causes for several inherited diseases that all show deformities in the skeletal system [68]. Moreover, several studies also showed that genetic defects in genes encoding the sulfatases disturb the sulfate metabolism and result in some human inherited diseases. For example, multiple sulfatase deficiency (MSD) is an inherited disease characterized by the deficiency of multiple sulfatases (OMIM 272200). Patients with MSD usually have deformities of the chest, problems with bone formation and growth and joint stiffness. To find out whether mutations in GAL3ST4 are also associated with the development of sporadic PE, we sequenced all the exons of GAL3ST4 in eight sporadic cases with PE and identified another missense mutation g.chr7: 99758263C $>\mathrm{T}$ in one of the patients (Supplementary information, Figure S1). This mutation has not been reported as a genetic polymorphism in the public databases (including the dbSNP132 and 1000 Genome Project databases) and is also absent from the 301 ethnicity-matched normal controls that we sequenced. However, mutation g.chr7: $99758263 \mathrm{C}>\mathrm{T}$ is located outside of the signal peptide region of Gal3ST-4 and the arginine residue affected by this mutation is less conservative than the one affected by mutation g.chr7: 99764688G $>$ A (Supplementary information, Figure S2). Thus, future studies are needed to investigate the exact roles of GAL3ST4 in the development of sporadic PE. Overall, our findings further confirmed that disturbance of the sulfate metabolism is likely to be a common cause for several inherited diseases with skeletal deformities.

Both cartilages and developing bones are abundant with various sulfated proteoglycans. The sulfate moieties provide a negative charge and are quite important for the structure and assembly of the skeletal system [9]. The sulfated proteoglycans are synthesized by different sulfotransferases, sulfatases and other related components in the Golgi body. The localization and organization of these active molecules in the Golgi apparatus are essential for the normal sulfation of the conjugated glycans, since the biosynthesis cascades are catalyzed by the stepwise enzyme reactions. Thus, it can be conceived that dysfunction of GAL3ST4 by genetic mutations will have a great effect on the sulfation of the various target glycoproteins. The four members of the Gal: $\rightarrow 3$-sulfotransferase family share 30\%-40\% amino acid sequence identity, but each of them shows distinct substrate specificities [5]. Although it has been shown that the Gal3ST-4 is highly specific for the core 1 structures generated in the O-linked glycans [10], future studies are needed to unravel the exact downstream targets or pathways of Gal3ST-4 that contribute to the development of familial PE.

Taken together, through whole-exome sequencing of a family with congenital $\mathrm{PE}$, we identified mutations in the sulfotransferase gene GAL3ST4 as the potential cause for PE. Our functional study further confirmed that genetic mutation of GAL3ST4 affects the functions of the encoded protein. To our knowledge, this is the first gene linked to the pathogenesis of PE. Our results along with data from previous studies implicate imbalance of sulfate metabolism as the common cause for several congenital skeletal deformities. These findings may lay the basis for future development of new diagnostic or therapeutic targets for PE or other conditions associated with abnormal development of cartilages and bones.

\section{Acknowledgments}

We would like to thank all the family members for their participation in the study. This study was mainly supported by grants from Shenzhen-Hongkong Innovative Projects (Experimental study of biological nano-artificial bone), Shenzhen Science and Technology Project (201001013) and the Promotion Program for Shenzhen Key Laboratory, Shenzhen, China (CXB200903090055A and CXB201005250016A to ZC), the National Natural Science Foundation of China (81101584), Shenzhen engineering lab of tumor clinical immune gene therapy and Shenzhen engineering lab of tumor genomic clinical application.

Song $\mathrm{Wu}^{1,2,3,4, *}$, Xiaojuan $\mathrm{Sun}^{1,4,5,{ }^{*}}$, Weimin $\mathrm{Zhu}$, , $^{1,}$ Yi Huang ${ }^{1,4, *}$, Lisha Mou, ${ }^{6, *}$, Mingming $\mathrm{Liu}^{7}, \mathrm{Xia} \mathrm{Li}^{7}$, Feiyang $\mathrm{Li}^{1}$, Xianxin $\mathrm{Li}^{6}$, Yong Zhang ${ }^{7}$, Zheng Wang ${ }^{8}$, Wencui $\mathrm{Li}^{1}$, Zesong $\mathrm{Li}^{1,4}$, Aifa Tang ${ }^{1,4}$, Yaoting Gui ${ }^{6}$, Rongfu Wang ${ }^{3}$, Weiping $\mathrm{Li}^{1}$, Zhiming Cai ${ }^{1,4}$,

Daping Wang ${ }^{1}$

${ }^{I}$ Shenzhen Second People's Hospital, The First Affiliated Hospital of Shenzhen University, Shenzhen, Guangdong 518035, China; ${ }^{2}$ Institute of Immunology, Zhongshan School 
of Medicine, Sun Yat-sen University, Guangzhou, Guangdong 510080, China; ${ }^{3}$ The Center for Cell and Gene Therapy, Baylor College of Medicine, Houston, TX 77030, USA; ${ }^{4}$ Shenzhen Key Laboratory of Genitourinary Tumor, Shenzhen Second People's Hospital, Shenzhen, Guangdong 518035, China; ${ }^{5}$ Shenzhen Engineering lab of Tumor Clinical Immune Gene Therapy, Shenzhen Second People's Hospital, Shenzhen, Guangdong, 518035, China; ${ }^{6}$ Guangdong and Shenzhen Key Laboratory of Male Reproductive Medicine and Genetics, Institute of Urology, Peking University Shenzhen Hospital, Shenzhen PKU-HKUST Medical Center, Shenzhen, Guangdong 518036, China; ${ }^{7}$ BGI-Shenzhen, Shenzhen, Guangdong 518083, China; ${ }^{8}$ Shenzhen First People's Hospital, Shenzhen, Guangdong 518001, China

*These five authors contributed equally to this work. Correspondence: Zhiming Cai ${ }^{\mathrm{a}}$, Daping Wang

a'E-mail: caizhiming2000@yahoo.com.cn

bE-mail: dapingwang1963@qq.com

\section{References}

1 Jaroszewski D, Notrica D, McMahon L, Steidley DE, Deschamps C. Current management of pectus excavatum: a review and update of therapy and treatment recommendations. $J \mathrm{Am}$ Board Fam Med 2010; 23:230-239.

2 Kotzot D, Schwabegger AH. Etiology of chest wall deformities - a genetic review for the treating physician. $J$ Pediatr Surg 2009; 44:2004-2011.
3 Creswick HA, Stacey MW, Kelly RE Jr, et al. Family study of the inheritance of pectus excavatum. J Pediatr Surg 2006; 41:1699-1703.

4 Gui Y, Guo G, Huang Y, et al. Frequent mutations of chromatin remodeling genes in transitional cell carcinoma of the bladder. Nat Genet 2011; 43:875-878.

5 Seko A, Hara-Kuge S, Yamashita K. Molecular cloning and characterization of a novel human galactose 3-O-sulfotransferase that transfers sulfate to $\mathrm{Gal} \beta 1 \rightarrow 3 \mathrm{GalNAc}$ residue in $O$ glycans. J Biol Chem 2001; 276:25697-25704.

6 Honke K, Taniguchi N. Sulfotransferases and sulfated oligosaccharides. Med Res Rev 2002; 22:637-654.

7 Superti-Furga A, Hastbacka J, Wilcox WR, et al. Achondrogenesis type IB is caused by mutations in the diastrophic dysplasia sulphate transporter gene. Nat Genet 1996; 12:100-102.

8 Kurima K, Warman ML, Krishnan S, et al. A member of a family of sulfate-activating enzymes causes murine brachymorphism. Proc Natl Acad Sci USA 1998; 95:8681-8685.

9 Heinegard D, Oldberg A. Structure and biology of cartilage and bone matrix noncollagenous macromolecules. FASEB $J$ 1989; 3:2042-2051.

10 Chandrasekaran EV, Lakhaman SS, Chawda R, Piskorz CF, Neelamegham S, Matta KL. Identification of physiologically relevant substrates for cloned Gal: 3-O-sulfotransferases (Gal3STs): distinct high affinity of Gal3ST-2 and LS180 sulfotransferase for the globo $\mathrm{H}$ backbone, Gal3ST-3 for N-glycan multiterminal Gal $\beta 1,4$ GlcNAc $\beta$ units and 6-sulfoGal $\beta 1$, $4 \mathrm{GlcNAc} \beta$, and Gal3ST-4 for the mucin core-2 trisaccharide. $J$ Biol Chem 2004; 279:10032-10041.

(Supplementary information is linked to the online version of the paper on the Cell Research website.) 